\section{Perfil de morbidade e gastos hospitalares com idosos no Paraná, Brasil, entre 2008 e 2012}

\author{
Morbidity profile and hospital expenses with elderly \\ patients in Paraná State, Brazil, 2008-2012
}

\section{Perfil de morbilidad y gastos hospitalarios con ancianos en Paraná, Brasil, entre 2008 y 2012}

Clarice da Luz Kernkamp ${ }^{1}$ Cassia Kely Favoretto Costa 2 Ely Mitie Massuda 3

Eraldo Schunk Silva 2 Mirian Ueda Yamaguchi 3 Marcelo Picinin Bernuci 3

\title{
Resumo
}

O aumento da população idosa leva a uma crescente demanda por serviços de saúde e expansão dos gastos, com consequências relevantes sobre a estabilidade econômica. Nesse contexto, o objetivo do estudo foi analisar o perfil das morbidades e gastos hospitalares com idosos em relação às condições socioeconômicas e demográficas do Paraná, Brasil, no período de 2008 a 2012, aplicando-se a análise fatorial em componentes principais e de agrupamentos. As regiões com maior e menor desenvolvimento econômico e humano apresentaram alta prevalência das internações e dos custos relativos às doenças do aparelho circulatório, respiratório e do coração para os sexos masculino e feminino. Já nas regiões com nível de desenvolvimento intermediário, as doenças do sistema nervoso em homens e do aparelho circulatório em mulheres se destacaram com relação às morbidades e gastos hospitalares. Assim, ações de prevenção e promoção de saúde a essa população idosa podem ser direcionadas para investigações regionais.

Envelhecimento da População; Idoso; Hospitalização; Análise Multivariada

\author{
1 Universidade Norte do \\ Paraná, Londrina, Brasil. \\ 2 Universidade Estadual de \\ Maringá, Maringá, Brasil. \\ 3 Centro de Ensino Superior \\ de Maringá, Maringá, Brasil. \\ Correspondência \\ C. K. F. Costa \\ Universidade Estadual de \\ Maringá. \\ Av. Colombo 5790, bloco C34, \\ sala 5, Maringá, $P R$ \\ 87020-900, Brasil. \\ cfavoretto@hotmail.com
}




\section{Introdução}

O crescimento do número de idosos em relação à população jovem e adulta, desde o limiar do século XXI, é característica marcante nos países em desenvolvimento, como o Brasil 1. Vivenciase uma transição no perfil demográfico da população com o aumento da expectativa de vida e redução das taxas de mortalidade e natalidade 2 . Os indivíduos idosos, com 60 anos ou mais, somam aproximadamente 21,7 milhões, representando $11 \%$ da população nacional total em 2010. As projeções indicam que em 2050 essa participação aumentará para $23,6 \%$, colocando o país entre os cinco maiores em contingente de idosos no mundo 3 .

No Paraná, Brasil, o crescimento da população idosa comparada aos jovens e adultos também é um fator de destaque. Em 2010, os indivíduos com 60 anos ou mais totalizaram 1,36 milhão, correspondendo a $11,2 \%$ da população total paranaense 2. Esse comportamento gera mudanças nos padrões econômicos e sociais do estado, uma vez que esse grupo permanecerá inserido no mercado de trabalho por mais tempo e demandará serviços de saúde apropriados e consequentemente mais custosos 4,5,6,7.

Reconhecidamente, o envelhecimento populacional está relacionado com o aumento da prevalência de doenças crônicas não transmissíveis, entre elas as cardiovasculares, pulmonares obstrutivas crônicas, do aparelho locomotor, endócrinas nutricionais e metabólicas, neoplasias e diabetes 8,9,10,11,12,13,14,15,16. Esse quadro gera maior demanda nas internações hospitalares, tratamentos medicamentosos e na reabilitação dos pacientes, o que induz ao aumento dos gastos nas atenções secundária e terciária do Sistema Único de Saúde (SUS) 17. No longo prazo, esse novo cenário impactará a sustentabilidade financeira do sistema de saúde e pode comprometer a oferta e qualidade dos serviços prestados à população 18,19.

A trajetória dos gastos com saúde sofre influências das diferenças demográficas, culturais e socioeconômicas que agem como atenuantes ou propulsores dos gastos 20,21 . Sob a ótica da atual transição populacional, quando as doenças crônicas tornam-se dominantes, a composição demográfica representa um aspecto relevante na análise de gastos com saúde 22 . Nesse sentido, a avaliação de custos tem sido uma das preocupações centrais dos gestores públicos, que buscam, com base em aspectos distintos das populações idosas de diferentes regiões, alocar de forma eficiente os recursos financeiros.

Considerando-se esse contexto, técnicas de estatística multivariada têm sido utilizadas para a determinação de fatores associados aos gastos em saúde 23,24,25,26. As metodologias análise de componentes principais (ACP) e análise de agrupamentos (cluster analysis) identificam padrões ou relações de comportamento entre variáveis, cujo conjunto de fatores resultantes permite identificar grupos homogêneos de comportamento por regiões geográficas em relação às diferentes variáveis consideradas no estudo $27,28,29,30,31$. Dessa forma, por meio dessa metodologia, o presente trabalho analisou o comportamento das morbidades e gastos hospitalares com idosos em relação às condições socioeconômicas e demográficas do Paraná, no período de 2008 a 2012.

\section{Material e método}

\section{Dados}

Trata-se de um estudo quantitativo, transversal, retrospectivo e analítico com enfoque em dados secundários do Sistema de Informações Hospitalares (SIH) do SUS. A área de abrangência dos dados correspondeu as 22 Regionais de Saúde do Paraná, sendo elas: Paranaguá, Curitiba e Região Metropolitana, Ponta Grossa, Irati, Guarapuava, União da Vitória, Pato Branco, Francisco Beltrão, Foz do Iguaçu, Cascavel, Campo Mourão, Umuarama, Cianorte, Paranavaí, Maringá, Apucarana, Londrina, Cornélio Procópio, Jacarezinho, Toledo, Telêmaco Borba e Ivaiporã. As regionais de saúde são unidades de administração de serviços de saúde, que estão localizadas com as proximidades regionais, ou metropolitanas, sendo instituídas com o propósito de facilitar o acesso à rede de saúde estadual (Secretaria da Saúde do Paraná. Regionais de saúde. http://www.saude.pr. gov.br/modules/conteudo/conteudo.php?con teudo=2752, acessado em 08/Mai/2014).

As variáveis de saúde utilizadas na pesquisa foram: (a) número de Autorizações de Internações Hospitalares (AIH) pagas pelo SUS; e (b) valor total destas internações. Essas informações são de domínio público gratuito e foram coletadas no SIH-SUS (Morbidade hospitalar do SUS - por local de residência - Paraná. http://tabnet.datasus.gov.br/cgi/deftohtm. exe?sih/cnv/nrPR.def, acessado em Jun/2013) para o período de 2008 a 2012. A coleta foi feita considerando-se as seguintes características: (1) geral, por local de residência do idoso; (2) sexo; (3) ano; e (4) capítulos de I a XX da Classificação Internacional de Doenças, 10 a revisão (CID-10), sendo: capítulos II: Neoplasias (tumores); IX: Doenças do aparelho circulatório; X: Doenças do aparelho respiratório; VI: Doen- 
ças do sistema nervoso, e as Doenças Cardíacas, tais como: reumática crônica do coração, hipertensão essencial (primária), outras doenças hipertensivas, Infarto agudo do miocárdio, outras doenças isquêmicas do coração.

Na pesquisa, utilizaram-se também dados demográficos (população de cada regional de saúde) e econômicos (Produto Interno Bruto PIB e Produto Interno Bruto per capita - PIB per capita dos municípios) coletados Na página eletrônica do Departamento de Informática do SUS (DATASUS. Informações de saúde (TABNET): demográficas e socioeconômicas. http://www2. datasus.gov.br/DATASUS/index.php?area=0206, acessado em Jun/2013), bem como fator social (Índice de Desenvolvimento Humano Municipal - IDH-M das cidades), que foi disponibilizado pelo Instituto Paranaense de Desenvolvimento Econômico e Social (IPARDES. Indicadores sociais. http://www.ipardes.gov.br/index.php?pg_conteu $\mathrm{do}=1 \&$ sistemas $=1 \&$ cod_sistema $=5 \&$ grupo_in dic $=2$, acessado em Jun/2013). A coleta das informações ocorreu entre junho e setembro de 2013.

Destaca-se que o PIB (em Reais) representa todos os bens e serviços finais produzidos pelos municípios do Paraná em determinado período. O PIB per capita (em Reais) corresponde ao valor médio agregado por individuo desses bens e serviços, ou seja, aborda a dimensão econômica de desenvolvimento. Já o IDH-M considera, além dessa dimensão (renda), a longevidade e a educação da região. $O$ índice varia de 0 a 1 , sendo que quanto mais próximo de 1 , maior o desenvolvimento humano 32.

A organização, tabulação e sumarização dos dados foram realizadas no Microsoft Excel 2010 (Microsoft Corp., Estados Unidos). Na sequência, utilizou-se o software SAS, versão 9.3 (SAS Inst., Cary, Estados Unidos), para a estimativa da ACP, de cluster e o estudo descritivo dos resultados por meio de tabelas de frequências cruzadas (simples e relativas).

\section{Método}

Para estabelecer grupos homogêneos de regionais de saúde em função do nível de desenvolvimento socioeconômico e demográfico, aplicou-se a metodologia de ACP e de cluster. No modelo ACP considerou-se como input uma matriz composta por 22 linhas, representando as regionais de saúde do Paraná e 13 colunas (Tabela 1), as quais referem-se às seguintes variáveis socioeconômicas e demográficas: $\mathrm{PIB}$ per capita de $2008\left(\mathrm{~V}_{1}\right)$, PIB per capita de $2009\left(\mathrm{~V}_{2}\right)$, PIB per capita de $2010\left(\mathrm{~V}_{3}\right)$, PIB de $2008\left(\mathrm{~V}_{4}\right)$, PIB de $2009\left(\mathrm{~V}_{5}\right)$, PIB de $2010\left(\mathrm{~V}_{6}\right)$, População de $2008\left(V_{7}\right)$, População de $2009\left(V_{8}\right)$, População de
$2010\left(\mathrm{~V}_{9}\right)$, População de $2011\left(\mathrm{~V}_{10}\right)$, População de $2012\left(\mathrm{~V}_{11}\right)$, IDH -M de $2000\left(\mathrm{~V}_{12}\right)$ e IDH-M de $2010\left(\mathrm{~V}_{13}\right)$.

A análise de agrupamentos foi realizada com base na matriz de escores gerados pela ACP. Após a determinação das áreas homogêneas de comportamento socioeconômico e demográfico, foi realizada a caracterização das morbidades e gastos para os respectivos grupos aplicando-se as tabelas de frequências cruzadas.

\section{Análise de componentes principais (ACP)}

A ACP, introduzida por Pearson em 1901 e por Hotelling em 1933 33, refere-se à técnica fatorial, cujo principal objetivo é a redução da dimensão da matriz de dados inicial. Por meio de combinações lineares das características iniciais criam-se novas variáveis, cujos resultados denominam-se "componentes principais".

Considerando-se $\mathrm{m}$ componentes e $\mathrm{p}$ variáveis $(m \leq p)$, tem-se a seguinte configuração de variáveis latentes, ou seja, componentes principais (CP) resultantes de combinações lineares perfeitas (sem erros) das variáveis iniciais 33,34,35:

$$
\begin{aligned}
& \mathrm{CP}_{1}=\mathrm{a}_{11} \mathrm{X}_{1}+\mathrm{a}_{2} \mathrm{X}_{2}+\ldots+\mathrm{ap}_{1} \mathrm{X}_{\mathrm{p}} \\
& \mathrm{CP}_{2}=\mathrm{a}_{12} \mathrm{X}_{1}+\mathrm{a}_{22} \mathrm{X}_{2}+\ldots+\mathrm{ap}_{2} \mathrm{X}_{\mathrm{p}} \\
& \begin{array}{lllll}
\cdots & \ldots & \ldots & \ldots
\end{array} \\
& \mathrm{CP}_{\mathrm{m}}=\mathrm{a}_{1 \mathrm{~m}} \mathrm{X}_{1}+\mathrm{a}_{22} \mathrm{X}_{2}+\ldots+\mathrm{ap}_{\mathrm{m}} \mathrm{X}_{\mathrm{p}}
\end{aligned}
$$

Na prática, a solução desse sistema de equações é única e considera a variação total presente no conjunto de variáveis iniciais, em que a primeira CP1 explica o máximo possível da variância dos dados iniciais, e a segunda CP2 explana o máximo possível da variância ainda não explicada e, assim por diante, até a última CPm que contribui com a menor parcela de explicação da variância total dos dados iniciais. Destaca-se que o critério para definir o número de componentes principais a reter no modelo refere-se aos fatores que apresentam autovalores $(\lambda \mathrm{i})$ maiores que uma unidade $(\lambda>1)$. Os fatores gerados, cujos autovalores são menores iguais a uma unidade $(\lambda i \leq 1)$, não explicam nem ao menos a variabilidade das variáveis retidas no fator 34,35 .

\section{Análise de agrupamentos (clusters)}

Os grupos homogêneos de regionais de saúde em função das características socioeconômicas e demográficas foram definidos baseando-se na cluster analysis. Nessa etapa, considera-se como matriz de input os escores gerados pela análise fatorial.

A análise de agrupamentos detecta o comportamento das variáveis por meio das seme- 
Tabela 1

Base de dados das Regionais de Saúde do Paraná, Brasil, 2008-2012.

\begin{tabular}{|c|c|c|c|c|c|c|c|c|c|c|c|c|c|}
\hline \multirow[t]{3}{*}{ Regional } & \multicolumn{3}{|c|}{ PIB per capita } & \multicolumn{3}{|c|}{ PIB } & \multicolumn{5}{|c|}{ População } & \\
\hline & & & 2010 & 2008 & 2009 & 2010 & 2008 & 2009 & 2010 & 2011 & 2012 & 2000 & 2010 \\
\hline & $\mathrm{V}_{1}$ & $\mathrm{~V}_{2}$ & $\mathrm{~V}_{3}$ & $\mathrm{~V}_{4}$ & $\mathrm{~V}_{5}$ & $\mathrm{v}_{6}$ & $v_{7}$ & $\mathrm{~V}_{8}$ & $\mathrm{~V}_{9}$ & $\mathrm{~V}_{10}$ & $\mathrm{~V}_{11}$ & $\mathrm{~V}_{12}$ & $\mathrm{~V}_{13}$ \\
\hline $\begin{array}{l}\text { Parana- } \\
\text { guá }\end{array}$ & $32.183,92$ & $25.585,57$ & $32.209,74$ & $8.212 .468,00$ & $6.573 .369,10$ & $8.548 .208,00$ & 255.173 & 256.917 & 265.392 & 267.664 & 269.858 & 0,65 & 0,75 \\
\hline $\begin{array}{l}\text { Metro- } \\
\text { politana } \\
\text { (Curitiba) }\end{array}$ & $22.907,21$ & $24.175,43$ & $29.466,32$ & $75.813 .579,70$ & 81.173 .3710 & $94.994 .580,00$ & 330.9594 & 335.7681 & 3.223 .836 & 3.255 .366 & 3.285 .851 & 0,75 & 0,82 \\
\hline $\begin{array}{l}\text { Ponta } \\
\text { Grossa }\end{array}$ & $15.807,97$ & $16.045,80$ & $18.268,55$ & $9.1531 .44,90$ & $9.381 .769,80$ & $10.512 .874,00$ & 579.021 & 584.687 & 575.463 & 579.815 & 584.017 & 0,62 & 0,76 \\
\hline Irati & $10.118,86$ & $9.673,35$ & $10.948,68$ & $1.627 .355,20$ & $1.566 .377,00$ & $1.762 .322,00$ & 160.824 & 161.927 & 160.962 & 161.900 & 162.804 & 0,63 & 0,73 \\
\hline $\begin{array}{l}\text { Guara- } \\
\text { puava }\end{array}$ & $10.961,88$ & $11.376,43$ & $12.891,18$ & $5.005 .894,00$ & $5.218 .154,30$ & $5.685 .913,00$ & 456.664 & 458.681 & 441.070 & 441.700 & 442.299 & 0,66 & 0,73 \\
\hline $\begin{array}{l}\text { União da } \\
\text { Vitória }\end{array}$ & $10.868,35$ & $10.581,46$ & $13.020,60$ & $1.840 .273,30$ & $1.805 .164,70$ & $2.152 .293,00$ & 169.324 & 170.597 & 165.299 & 166.057 & 166.788 & 0,72 & 0,74 \\
\hline $\begin{array}{l}\text { Pato } \\
\text { Branco }\end{array}$ & $15.898,50$ & $16.656,72$ & $18.666,84$ & $3.972 .287,60$ & $4.182 .620,00$ & $4.662 .846,00$ & 249.853 & 251.107 & 249.793 & 250.898 & 251.960 & 0,68 & 0,78 \\
\hline $\begin{array}{l}\text { Fran- } \\
\text { cisco }\end{array}$ & $11.646,52$ & $12.873,05$ & $14.200,99$ & $3.897 .589,70$ & $4.320 .710,50$ & $4.795 .716,00$ & 334.657 & 335.640 & 337.703 & 338.927 & 340.093 & 0,66 & 0,78 \\
\hline Beltrã & & & & & & & & & & & & & \\
\hline $\begin{array}{l}\text { Foz do } \\
\text { Iguaçu }\end{array}$ & $17.759,65$ & $18.837,78$ & $22.850,52$ & $7.994 .792,60$ & $8.609 .410,20$ & $8.884 .170,00$ & 450.166 & 457.029 & 388.795 & 389.522 & 390.220 & 0,66 & 0,75 \\
\hline Cascavel & 15124,93 & $16.184,17$ & $17.828,25$ & $7.780 .084,70$ & $8.391 .509,40$ & $9.045 .788,00$ & 514.388 & 518.501 & 507.385 & 510.281 & 513.071 & 0,69 & 0,78 \\
\hline $\begin{array}{l}\text { Campo } \\
\text { Mourão }\end{array}$ & $14.217,01$ & $14.329,88$ & $15.430,14$ & $4.824 .129,60$ & $4.847 .140,70$ & $5.155 .595,00$ & 339.321 & 338.254 & 334.125 & 333.177 & 332.246 & 0,68 & 0,76 \\
\hline $\begin{array}{l}\text { Umuara- } \\
\text { ma }\end{array}$ & $10.138,03$ & $11.380,16$ & $13.886,27$ & $2.685 .127,60$ & $3.019 .395,90$ & $3.681 .139,00$ & 264.857 & 265.321 & 265.092 & 265.648 & 266.174 & 0,68 & 0,76 \\
\hline Cianorte & $12.461,51$ & $14.715,87$ & $17.021,35$ & $1.756 .200,50$ & $2.095 .995,40$ & $2.424 .402,00$ & 140.930 & 142.431 & 142.433 & 143.758 & 145.037 & 0,64 & 0,76 \\
\hline $\begin{array}{l}\text { Parana- } \\
\text { vaí }\end{array}$ & $9.747,43$ & $10.789,53$ & $12.468,91$ & $2.528 .258,10$ & $2.807 .230,30$ & $3.248 .699,00$ & 259.377 & 260.181 & 260.544 & 261.462 & 262.333 & 0,69 & 0,76 \\
\hline Maringá & $13.964,70$ & $15.986,55$ & $17.840,56$ & $9.855 .835,50$ & $11.392 .384,00$ & $13.084 .335,00$ & 705.768 & 712.623 & 733.404 & 741.222 & 748.686 & 0,74 & 0,81 \\
\hline $\begin{array}{l}\text { Apuca- } \\
\text { rana }\end{array}$ & $12.785,92$ & $14.088,88$ & $15.557,19$ & $4.392 .025,70$ & $4.880 .065,40$ & $5.397 .908,00$ & 343.505 & 346.377 & 346.972 & 349.598 & 352.126 & 0,67 & 0,75 \\
\hline Londrina & $15.014,52$ & $16.473,76$ & $18.141,2$ & $13.008 .861,30$ & $14.396 .384,00$ & $15.805 .832,00$ & 866.419 & 873.898 & 871.267 & 877.720 & 884.039 & 0,72 & 0,79 \\
\hline $\begin{array}{l}\text { Cornélio } \\
\text { Procópio }\end{array}$ & $10.763,31$ & $10.807,83$ & $12.623,81$ & $2.507 .926,70$ & $2.515 .014,40$ & $2.852 .489,00$ & 233.007 & 232.703 & 225.961 & 225.335 & 224.721 & 0,69 & 0,76 \\
\hline $\begin{array}{l}\text { Jacarezi- } \\
\text { nho }\end{array}$ & $9.310,60$ & $10.101,8$ & $11.995,67$ & $2.609 .407,30$ & $2.837 .726,30$ & $3.336 .126,00$ & 280.262 & 280.913 & 278.111 & 278.615 & 279.093 & 0,64 & 0,74 \\
\hline Toledo & $17.735,86$ & $17.938,66$ & $19.917,99$ & $6.279 .700,90$ & $6.404 .926,90$ & $7.143 .785,00$ & 354.068 & 357.046 & 358.660 & 361.473 & 364.184 & 0,69 & 0,77 \\
\hline $\begin{array}{l}\text { Telêma- } \\
\text { co Borba }\end{array}$ & $12.767,05$ & $12.736,92$ & $15.701,93$ & $2.244 .485,20$ & $2.258 .637,80$ & $2.715 .209,00$ & 175.803 & 177.330 & 172.922 & 173.974 & 174.986 & 0,64 & 0,73 \\
\hline Ivaiporã & $8.653,97$ & $8.980,44$ & $10.043,63$ & $1.273 .761,20$ & $1.314 .592,20$ & $1.399 .449,00$ & 147.188 & 146.384 & 139.337 & 138.237 & 137.169 & 0,62 & 0,73 \\
\hline
\end{tabular}

IDH-M: Índice de Desenvolvimento Humano Municipal; PIB: Produto Interno Bruto; V 1 : PIB per capita de 2008; V 2 PIB per capita de 2009; V de 2010; $V_{4}$ : PIB de 2008; $V_{5}$ : PIB de 2009; $V_{6}$ : PIB de 2010; $V_{7}$ : População de 2008; $V_{8}$ : População de 2009; $\bigvee_{9}$ : População de 2010; $\bigvee_{10}$ : População de 2011; $V_{11}$ : População de 2012; $V_{12}$ : IDH-M de 2000; $V_{13}$ : IDH-M de 2010.

lhanças ou dissimilaridades entre elas $34,35,36$. Para isso, deve-se aplicar o critério de classificação hierárquica de Ward com distância Euclidiana, que não exige alguma condição nos dados, pois não há a necessidade de se definir uma relação causal entre as variáveis utilizadas. Nesse sentido, essa abordagem não faz uso de modelos aleatórios, sendo simplesmente útil para sintetizar um conjunto de dados. Porém, é crucial notar que a forma de trabalhar com os dados (brutos, normalizados, centrados), a definição da medida de distância entre os grupos e o critério de 
aglomeração dos objetos podem influenciar nos resultados da análise 34,35 .

A estratégia de proceder à análise de agrupamentos, após a ACP, apresenta duas grandes vantagens. Primeiro, a análise de agrupamento é mais especificamente adaptada para construir agrupamentos (dendogramas). Segundo, após a aplicação da ACP elimina-se a multicolinearidade dos dados 35 .

\section{Resultados}

\section{Análise de componentes principais (ACP)}

Na Tabela 2, estão os resultados da análise fatorial, em que foram retidos apenas os fatores que apresentaram autovalores maiores que uma unidade, conforme já destacado na metodologia. Adotando-se esse critério retiveram-se dois fatores, em que o primeiro autovalor $(\lambda 1=9,89)$ explica $76,08 \%$ da variabilidade dos dados. O segundo autovalor $(\lambda 2=1,92)$ explana $14,8 \%$, totalizando $90,88 \%$ de variabilidade explicada (VE) para os dados (Tabela 2).

Após a análise das cargas fatoriais (não mostrada aqui) verificou-se que o Fator 1 é formado pelas variáveis PIB 2008, PIB 2009, PIB 2010, População 2008, População 2009, População 2010, População 2011, População 2012, IDH 2000 e IDH 2010. Já o Fator 2 é composto pelas variáveis PIB per capita 2008, PIB per capita 2009 e PIB per capita 2010.

O foco aqui não é fazer a interpretação dos fatores retidos e sim utilizar a análise fatorial para obter os escores a serem utilizados no cluster. A vantagem de conjugar essas duas técnicas é que se obtém ao final a variabilidade dos dados explicada em termos de fatores ortogonais (não correlacionados). Caso o cluster tivesse sido aplicada nos dados originais, seria preciso contornar o problema da colinearidade entre as variáveis. Assim, na Tabela 3 estão os resultados dos fatores 1 e 2, ao relacionar as 22 regionais de saúde e as variáveis socioeconômicas e demográficas abordadas neste estudo.

\section{Análise de agrupamento (clusters)}

Retidos os escores fatoriais para as 22 regionais de saúde procedeu-se aos clusters considerando os escores da Tabela 3 como "variáveis inputs". O dendrograma obtido com base na matriz de dados tendo como variáveis inputs os escores dos dois primeiros fatores retidos, sugere a formação de seis grupos homogêneos, cada um refletindo um tipo de estrutura demográfica e nível de desenvolvimento socioeconômico (Figura 1). Esses grupos foram obtidos utilizando-se como medida de dissimilaridade a Distância Euclidiana Simples e como algoritmo de aglomeração o método de Ward.

Nessa linha, o Paraná está subdivido nos seguintes grupos (Tabela 4): (a) Grupo I, composto pela regional de Curitiba (Região Metropolitana); (b) Grupo II (áreas do centro/norte/noroeste/sudeste do estado), formado pelas regionais de $\mathrm{Pa}$ ranavaí, Jacarezinho, Cornélio Procópio, União da Vitória, Umuarama, Guarapuava, Ivaiporã e Irati; (c) Grupo III (norte pioneiro), regionais de Londrina e Maringá; (d) Grupo IV (região central), com as regionais de Telêmaco Borba, Cianorte, Apucarana, Campo Mourão e Francisco Beltrão; (e) Grupo V (oeste/sudoeste), com Foz do Iguaçu, Cascavel, Toledo, Pato Branco e Ponta Grossa; e (d) Grupo VI (leste), constituído pela regional de Paranaguá.

Analisando o comportamento homogêneo quanto às condições socioeconômicas e demográficas das regionais, observa-se na Tabela 4 que o Grupo I apresentou o 20 maior PIB per capita do estado (R\$29.466,32) e o maior IDH-M $(0,82)$ para 2010. O Grupo II registrou o menor PIB per capita para o mesmo ano, que variou entre o mínimo de $\mathrm{R} \$ 8.980,44$ e o máximo de $\mathrm{R} \$ 11.380,16$. Já o agrupamento III demonstrou o PIB per capita em torno de R\$18.000,00 e o 2 o maior IDH-M do estado (entre 0,79 e 0,81).

O Grupo IV (Tabela 4) demonstrou níveis intermediários de PIB per capita quando comparado às demais áreas agrupadas, com valor mínimo de $\mathrm{R} \$ 14.200,99$ e máximo de $\mathrm{R} \$ 17.021,35$. Os índices de desenvolvimento humano foram sa-

Tabela 2

Autovalores e variância explicada para os três primeiros fatores retidos.

\begin{tabular}{lccc}
\hline Fatores retidos & Autovalores & Variância explicada (\%) & $\begin{array}{c}\text { Variância explicada } \\
\text { acumulada (\%) }\end{array}$ \\
\hline 1 & 9,890316 & 76,08 & 76,08 \\
2 & 1,923973 & 14,80 & 90,88 \\
\hline
\end{tabular}


Escores fatoriais para as 22 Regionais de Saúde do Paraná, Brasil, gerados com base nas 13 variáveis socioeconômicas e demográficas consideradas no estudo.

\begin{tabular}{|c|c|c|c|}
\hline Regional de saúde & Código & Fator 1 & Fator 2 \\
\hline Paranaguá & pn & $-0,30592$ & 3,660010 \\
\hline Metropolitana & $\mathrm{mt}$ & $-4,14906$ & $-0,891887$ \\
\hline Ponta Grossa & pg & $-0,05828$ & 0,479051 \\
\hline Irati & ir & 0,74031 & $-0,616632$ \\
\hline Guarapuava & $g u$ & 0,33258 & $-0,644967$ \\
\hline União da Vitória & uv & 0,49622 & $-0,677677$ \\
\hline Pato Branco & $\mathrm{pb}$ & 0,09935 & 0,599112 \\
\hline Francisco Beltrão & $\mathrm{fb}$ & 0,22594 & $-0,340034$ \\
\hline Foz do Iguaçu & $\mathrm{fi}$ & $-0,08069$ & 1,143088 \\
\hline Cascavel & $\mathrm{ca}$ & $-0,15901$ & 0,172606 \\
\hline Campo Mourão & $\mathrm{cm}$ & 0,20949 & 0,042862 \\
\hline Umuarama & um & 0,38605 & $-0,579522$ \\
\hline Cianorte & ci & 0,46479 & 0,347847 \\
\hline Paranavaí & pa & 0,39954 & $-0,793902$ \\
\hline Maringá & ma & $-0,51804$ & $-0,329655$ \\
\hline Apucarana & ap & 0,25651 & $-0,060140$ \\
\hline Londrina & lo & $-0,60285$ & $-0,216641$ \\
\hline Cornélio Procópio & $\mathrm{cp}$ & 0,43177 & $-0,659744$ \\
\hline Jacarezinho & ja & 0,54821 & $-0,733169$ \\
\hline Toledo & to & $-0,05698$ & 0,802243 \\
\hline Telêmaco Borba & tb & 0,53512 & 0,096536 \\
\hline Ivaiporã & iv & 0,80496 & $-0,799385$ \\
\hline
\end{tabular}

tisfatórios (IDH-M entre 0,76 e 0,78), pois estão próximos a 1.

O Grupo V apresentou IDH-M entre 0,75 e 0,78 , todavia com nível de PIB per capita que variou entre $\mathrm{R} \$ 17.828,25$ e $\mathrm{R} \$ 22.859,22$ (Tabela 4). Por fim, o Grupo VI registrou o maior PIB per capita para o ano de 2010 dentre aqueles analisados e um dos IDH-M mais baixos $(0,75)$.

\section{Perfil das morbidades e gastos hospitalares com idosos}

Na Tabela 5, observa-se que entre 2008 e 2012 ocorreram no Paraná 878.377 internações, com concentração maior nos grupos IV e II (participações de 29,09\% e 23,16\%, nesta ordem). O Grupo VI demandou um menor número de internações de idosos, representando apenas 1,46\% do total.

Na análise do Grupo I verifica-se a ocorrência de 159.478 internações, sendo $50,1 \%$ deste total composto por homens e $49,9 \%$ por mulheres. A maior prevalência de internações aconteceu devido às doenças do aparelho circulatório e do coração para ambos os sexos, sendo de $36,38 \%$ e $30,16 \%$ para o masculino e de $35,99 \%$ e $29,1 \%$ para o feminino, respectivamente. No Grupo II, o total de internações correspondeu a 203.389 casos, em que $50,52 \%$ foram de homens, com prevalência mais alta das doenças circulatórias $(35,75 \%)$ e respiratórias $(27,71 \%)$. As mulheres foram responsáveis por $49,48 \%$ do total, sendo que as doenças circulatórias $(37,09 \%)$ e do coração $(27,36 \%)$ apresentaram o maior destaque.

Para o Grupo III, observou-se que do total de 111.312 internações, $51,57 \%$ foram de idosos, cujas doenças que mais acometeram o sexo masculino foram as circulatórias $(31,81 \%)$ e respiratórias $(25,18 \%)$. As idosas participaram com $48,54 \%$ deste total, com uma frequência de $33,32 \%$ e $25,83 \%$ para as mesmas doenças, nesta ordem. Já no Grupo IV, de um total de 89.125 homens, $27,56 \%$ deles foram internados por doenças do sistema nervoso $(27,56 \%)$, seguidas do aparelho circulatório (21,36\%). As mulheres foram acometidas em $73,73 \%$ pelas doenças do aparelho circulatório (Tabela 5).

De um total de 66.073 homens internados (Grupo V), 34,87\% e 28,13\% deles demonstra- 
Figura 1

Agrupamento de 22 Regionais de Saúde do Paraná, Brasil - método de Ward - Distância Euclidiana Simples.

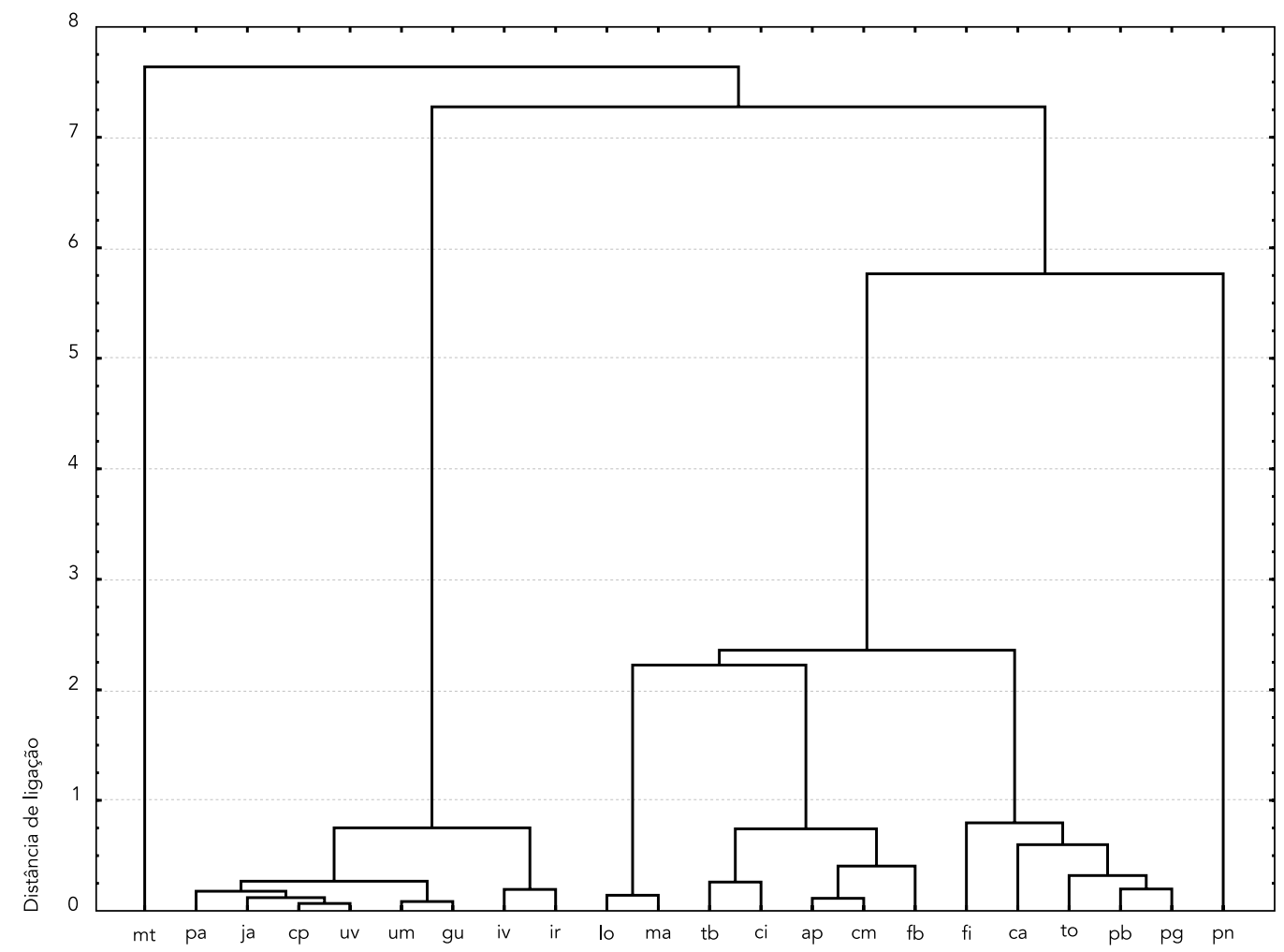

ap: Apucarana; ca: Cascavel; ci: Cianorte; cm: Campo Mourão; cp: Cornélio Procópio; fb: Francisco Beltrão; fi: Foz do lguaçu; gu: Guarapuava; ir: Irati; iv: Ivaiporã; ja: Jacarezinho; lo: Londrina; ma: Maringá; mt: Metropolitana; pa: Paranavaí; pb: Pato Branco; pg: Ponta Grossa; pn: Paranaguá; tb: Telêmaco Borba; to: Toledo; um: Umuarama; uv: União da Vitória.

Tabela 4

Agrupamentos de Regionais de Saúde do Paraná, Brasil.

\begin{tabular}{|c|c|c|c|c|c|}
\hline \multirow[t]{2}{*}{ Grupos } & \multirow[t]{2}{*}{ Regionais de Saúde } & \multicolumn{2}{|c|}{ IDH (2010) } & \multicolumn{2}{|c|}{ Cidade com menor/maior PIB per capita 2010 (R\$) } \\
\hline & & Menor & Maior & Menor & Maior \\
\hline Grupo I & Curitiba e Região Metropolitana & \multicolumn{2}{|c|}{0,82 * } & \multicolumn{2}{|c|}{$29.466,32$ * } \\
\hline Grupo ॥ & $\begin{array}{c}\text { Paranavaí, Jacarezinho, Cornélio Procópio, União Vitória, } \\
\text { Umuarama, Guarapuava, Ivaiporã, Irati }\end{array}$ & 0,74 & 0,76 & $8.980,44$ (Jacarezinho) & $11.380,16$ (Umuarama) \\
\hline Grupo III & Londrina, Maringá & 0,79 & 0,81 & 17.840,56 (Maringá) & $18.141,20$ (Londrina) \\
\hline Grupo IV & $\begin{array}{l}\text { Telêmaco Borba, Cianorte, Apucarana, Campo Mourão, } \\
\text { Francisco Beltrão }\end{array}$ & 0,76 & 0,78 & 14.200,99 (Francisco Beltrão) & $17.021,35$ (Cianorte) \\
\hline Grupo V & Foz do Iguaçu, Cascavel, Toledo, Pato Branco, Ponta Grossa & 0,75 & 0,78 & $17.828,25$ (Cascavel) & $22.850,22$ (Foz do Iguaçu) \\
\hline Grupo VI & Paranaguá & \multicolumn{2}{|c|}{0,75 * } & \multicolumn{2}{|c|}{$32.209,74$ * } \\
\hline
\end{tabular}

IDH: Índice de Desenvolvimento Humano; PIB: Produto Interno Bruto.

* Valores agregados. 
Tabela 5

Frequência das internações hospitalares do Sistema Único de Saúde (SUS) com idosos por gênero, Classificação Internacional de Doenças, 10 ạ revisão (CID-10) e grupos socioeconômicos. Paraná, Brasil, 2008-2012.

\begin{tabular}{|c|c|c|c|c|c|c|c|c|c|c|c|c|}
\hline \multirow[t]{2}{*}{ CID-10 } & \multicolumn{2}{|c|}{ Grupo I } & \multicolumn{2}{|c|}{ Grupo II } & \multicolumn{2}{|c|}{ Grupo III } & \multicolumn{2}{|c|}{ Grupo IV } & \multicolumn{2}{|c|}{ Grupo V } & \multicolumn{2}{|c|}{ Grupo VI } \\
\hline & $\mathbf{n}$ & $\%$ & $\mathbf{n}$ & $\%$ & $\mathbf{n}$ & $\%$ & $\mathbf{n}$ & $\%$ & $\mathbf{n}$ & $\%$ & $\mathbf{n}$ & $\%$ \\
\hline \multicolumn{13}{|l|}{ Masculino } \\
\hline $\begin{array}{l}\text { IX. Doenças do aparelho } \\
\text { circulatório }\end{array}$ & 29.072 & 36,38 & 36.727 & 35,75 & 18.259 & 31,81 & 19.036 & 21,36 & 18.587 & 28,13 & 3.077 & 45,22 \\
\hline Doenças do coração & 24.098 & 30,16 & 26.479 & 25,77 & 12.810 & 22,31 & 16.552 & 18,57 & 13.790 & 20,87 & 1.733 & 25,47 \\
\hline $\begin{array}{l}\text { X. Doenças do aparelho } \\
\text { respiratório }\end{array}$ & 13.409 & 16,78 & 28.470 & 27,71 & 14.457 & 25,18 & 15.280 & 17,14 & 23.038 & 34,87 & 1.213 & 17,83 \\
\hline II. Neoplasias (tumores) & 11.981 & 14,99 & 8.203 & 7,98 & 9.562 & 16,66 & 13.697 & 15,37 & 8.625 & 13,05 & 684 & 10,05 \\
\hline VI. Doenças do sistema nervoso & 1.345 & 1,68 & 2.868 & 2,79 & 2.319 & 4,04 & 24.560 & 27,56 & 2.033 & 3,08 & 97 & 1,43 \\
\hline Total & 79.905 & 100,00 & 102.747 & 100,00 & 57.407 & 100,00 & 89.125 & 100,00 & 66.073 & 100,00 & 6.804 & 100,00 \\
\hline \multicolumn{13}{|l|}{ Feminino } \\
\hline $\begin{array}{l}\text { IX. Doenças do aparelho } \\
\text { circulatório }\end{array}$ & 28.639 & 35,99 & 37.326 & 37,09 & 17.960 & 33,32 & 122.685 & 73,73 & 17.928 & 25,68 & 2.517 & 42,03 \\
\hline Doenças do coração & 23.153 & 29,10 & 27.538 & 27,36 & 12.404 & 23,01 & 12.659 & 7,61 & 13.595 & 19,47 & 1.508 & 25,18 \\
\hline $\begin{array}{l}\text { X. Doenças do aparelho } \\
\text { respiratório }\end{array}$ & 15.109 & 18,99 & 27.381 & 27,21 & 13.926 & 25,83 & 12.578 & 7,56 & 23.803 & 34,10 & 1.241 & 20,72 \\
\hline II. Neoplasias (tumores) & 11.340 & 14,25 & 6.175 & 6,14 & 7.581 & 14,06 & 8.336 & 5,01 & 12.412 & 17,78 & 564 & 9,42 \\
\hline VI. Doenças do sistema nervoso & 1.332 & 1,67 & 2.222 & 2,21 & 2.034 & 3,77 & 10.138 & 6,09 & 2.073 & 2,97 & 159 & 2,65 \\
\hline Total & 79.573 & 100,00 & 100.642 & 100,00 & 53.905 & 100,00 & 166.396 & 100,00 & 69.811 & 100,00 & 5.989 & 100,00 \\
\hline Total geral & 159.478 & & 203.389 & & 111.312 & & 255.521 & & 135.884 & & 12.793 & \\
\hline
\end{tabular}

ram problemas no aparelho respiratório e circulatório, respectivamente. A mesma realidade dessas doenças foi observada para o número de mulheres (69.811) com participações de 34,1\% e $25,68 \%$, nesta ordem. Por fim, o Grupo VI totalizou 12.793 internações, com participação de $53,19 \%$ dos homens e $46,81 \%$ das mulheres. O CID-10 das doenças do aparelho circulatório foi o que mais prevaleceu para ambos os sexos $(45,22 \%$ para os homens e $42,03 \%$ para mulheres). Em segundo lugar na classificação estão as doenças do coração com participação de 25,47\% para indivíduos do sexo masculino e 25,18\% para o feminino (Tabela 5).

Em termos de gastos hospitalares, verificase que o SUS apresentou um custo de 2.769,74 milhões de Reais com internações de idosos no Paraná, entre 2008 e 2012 (Tabela 6). Nesse total, o Grupo III apresentou a maior participação $(51,05 \%)$ e o Grupo VI, a menor $(1,13 \%)$.

Observa-se que as internações de homens ( $\mathrm{R} \$ 235,59$ milhões) geraram uma maior demanda de recursos financeiros no Grupo I, sendo que as doenças do coração foram responsáveis por $42,01 \%$ deste total, seguidas do aparelho circulatório $(40,4 \%)$. As mesmas doenças gera- ram um custo nominal de $\mathrm{R} \$ 77,35$ milhões e $\mathrm{R} \$$ 73,75 milhões com as mulheres, respectivamente (Tabela 6).

No Grupo II, o SUS apresentou os maiores gastos com doenças do aparelho circulatório e do coração em homens (participação em torno de $43,53 \%$ e $33,81 \%$, respectivamente) e mulheres (frequência de $43,37 \%$ e 32,95\%, nesta ordem)

A concentração mais alta de gastos do SUS no Grupo III, sexo masculino, ocorreu com as doenças do aparelho circulatório, correspondendo a $28,14 \%$ do total de 108,61 milhões de Reais (Tabela 6). Em segundo lugar estão as doenças do sistema nervoso, com 24,93\%. Para o sexo feminino, o governo gastou $27,8 \%$ do total de $\mathrm{R} \$$ $1.305,41$ milhão com neoplasias, seguidas das doenças do aparelho respiratório (26,57\%).

O Grupo IV demandou um custo total de 412,96 milhões de Reais, sendo que 72,26\% deste total foram direcionados às idosas atendidas pelo SUS, em que os problemas do aparelho circulatório foram o destaque no período $(62,89 \%$ do total de R\$298,42 milhões). No caso dos homens, as doenças do sistema nervoso (25,34\%) e do aparelho circulatório (22,52\%) registraram as maiores participações. 
Gastos totais nominais (em milhões de Reais) do Sistema Único de Saúde (SUS) com morbidades hospitalares em idosos, por gênero, Classificação Internacional de Doenças, 10ạ revisão (CID-10) e grupo socioeconômico. Paraná, Brasil, 2008-2012.

\begin{tabular}{|c|c|c|c|c|c|c|c|c|c|c|c|c|}
\hline \multirow[t]{2}{*}{ CID-10 } & \multicolumn{2}{|c|}{ Grupo I } & \multicolumn{2}{|c|}{ Grupo II } & \multicolumn{2}{|c|}{ Grupo III } & \multicolumn{2}{|c|}{ Grupo IV } & \multicolumn{2}{|c|}{ Grupo V } & \multicolumn{2}{|c|}{ Grupo VI } \\
\hline & Valor (R\$) & $\%$ & Valor (R\$) & $\%$ & Valor (R\$) & $\%$ & Valor (R\$) & $\%$ & Valor (R\$) & $\%$ & Valor (R\$) & $\%$ \\
\hline \multicolumn{13}{|l|}{ Masculino } \\
\hline $\begin{array}{l}\text { IX. Doenças do aparelho } \\
\text { circulatório }\end{array}$ & 95,19 & 40,40 & 70,37 & 43,53 & 30,56 & 28,14 & 25,79 & 22,52 & 40,71 & 39,27 & 7,91 & 52,91 \\
\hline Doenças do coração & 98,97 & 42,01 & 54,66 & 33,81 & 21,56 & 19,85 & 24,54 & 21,42 & 29,05 & 28,02 & 4,91 & 32,84 \\
\hline $\begin{array}{l}\text { X. Doenças do aparelho } \\
\text { respiratório }\end{array}$ & 21,31 & 9,05 & 22,14 & 13,70 & 16,89 & 15,55 & 19,25 & 16,81 & 19,57 & 18,88 & 1,00 & 6,69 \\
\hline II. Neoplasias (tumores) & 17,87 & 7,59 & 10,92 & 6,75 & 12,53 & 11,54 & 15,93 & 13,91 & 11,84 & 11,42 & 1,00 & 6,69 \\
\hline $\begin{array}{l}\text { VI. Doenças do sistema } \\
\text { nervoso }\end{array}$ & 2,25 & 0,96 & 3,58 & 2,21 & 27,07 & 24,92 & 29,03 & 25,34 & 2,50 & 2,41 & 0,12 & 0,80 \\
\hline Total & 235,59 & 100,00 & 161,66 & 100,00 & 108,61 & 100,00 & 114,54 & 100,00 & 103,66 & 100,00 & 14,95 & 100,00 \\
\hline \multicolumn{13}{|l|}{ Feminino } \\
\hline $\begin{array}{l}\text { IX. Doenças do aparelho } \\
\text { circulatório }\end{array}$ & 77,35 & 40,61 & 56,49 & 43,38 & 218,95 & 16,77 & 187,68 & 62,89 & 30,39 & 33,83 & 8,49 & 51,83 \\
\hline Doenças do coração & 73,75 & 38,72 & 42,91 & 32,95 & 206,50 & 15,82 & 43,09 & 14,44 & 21,83 & 24,30 & 5,25 & 32,05 \\
\hline $\begin{array}{l}\text { X. Doenças do aparelho } \\
\text { respiratório }\end{array}$ & 21,82 & 11,46 & 18,63 & 14,31 & 346,87 & 26,57 & 23,14 & 7,75 & 19,91 & 22,16 & 1,66 & 10,13 \\
\hline II. Neoplasias (tumores) & 15,65 & 8,22 & 7,59 & 5,83 & 362,90 & 27,80 & 17,14 & 5,74 & 15,54 & 17,30 & 0,72 & 4,40 \\
\hline $\begin{array}{l}\text { VI. Doenças do sistema } \\
\text { nervoso }\end{array}$ & 1,89 & 0,99 & 4,61 & 3,54 & 170,19 & 13,04 & 27,37 & 9,17 & 2,16 & 2,40 & 0,26 & 1,59 \\
\hline Total & 190,46 & 100,00 & 130,23 & 100,00 & $1.305,41$ & 100,00 & 298,42 & 100,00 & 89,83 & 100,00 & 16,38 & 100,00 \\
\hline Total geral & 426,05 & & 291,89 & & $1.414,02$ & & 412,96 & & 193,49 & & 31,33 & \\
\hline
\end{tabular}

As doenças do aparelho circulatório e do coração foram responsáveis pelos maiores gastos do SUS nos Grupos V e VI (Tabela 6). No Grupo $\mathrm{V}$, sexo masculino, essas doenças apresentaram uma participação relativa de 39,27\% e 28,02\% do total de 103,66 milhões de Reais, respectivamente. Já para o sexo feminino, os percentuais foram de $33,83 \%$ e $24,3 \%$ de um montante de $\mathrm{R} \$ 89,83$ milhões, nesta ordem. No Grupo VI (categoria dos homens), 52,91\% do total de 14,95 milhões de Reais foram com doenças do aparelho circulatório e 32,84\% dos gastos com problemas do coração. Em mulheres, de um total de R\$ 16,38 milhões, $51,83 \%$ e $32,05 \%$ deles foram para as mesmas doenças.

\section{Discussão}

Os resultados deste estudo permitiram verificar que no Paraná existem diferenças no comportamento das morbidades e dos custos hospitalares de idosos em função das condições socioeconômicas e demográficas de cada região. As regionais de Curitiba (Região Metropolitana - Grupo
I), de Londrina e Maringá (Grupo III) estão situadas nas áreas mais desenvolvidas do estado, apresentando maior densidade demográfica e dimensão econômica (representadas pelos valores altos do PIB per capita) e melhor índice de desenvolvimento humano (o valor do IDH-M de cada uma delas está mais próximo a 1). A possível influência desses determinantes no Grupo I foi maior nas morbidades e gastos hospitalares com doenças do aparelho circulatório e do coração para homens e mulheres. No Grupo III, a associação desses fatores foi evidenciada entre as internações dos aparelhos circulatório e respiratório. Contudo, esse agrupamento apresentou gastos nominais com neoplasias, sexo feminino, muito acima da média das demais regionais de saúde do Paraná.

Berentein \&Wajnman 22 compararam o efeito da composição etária da população sobre os gastos com internação hospitalar para duas regiões metropolitanas (Curitiba, Paraná e Belém, Pará) do Brasil em 2004. Dentre os principais resultados, constataram que para Curitiba os gastos médios de internação tendem a ser mais altos nas idades mais avançadas, o que não foi obser- 
vado em Belém. Concluíram que esse fato pode ocorrer, pois a cidade paranaense apresenta grau mais elevado de desenvolvimento econômico, melhor oferta de serviços de saúde para os idosos (tecnologia mais avançada, profissionais mais qualificados, infraestrutura hospitalar com qualidade, entre outros) e uma população mais envelhecida do que a outra cidade.

A população idosa no Brasil, entre 2002 e 2011, foi responsável por $27,85 \%$ das internações hospitalares e $36,47 \%$ dos recursos pagos por elas. Nesse período, as doenças dos aparelhos circulatório e respiratório foram responsáveis pelas maiores taxas de internação para homens $(45,01 \%$ e $29,85 \%)$ e para mulheres $(37,92 \%$ e $22,8 \%$, respectivamente). Além disso, representaram os maiores índices de custo em reais por pessoa 37 . Esse fato também foi confirmado em pesquisa 16 sobre a situação de saúde dos idosos da Região Metropolitana de São Paulo, que apresentou as doenças dos aparelhos circulatório e respiratório como as mais frequentes neste grupo, respectivamente.

Castro et al. ${ }^{38}$, por sua vez, analisaram o perfil das morbidades hospitalares de idosos das Regionais de Saúde do Paraná para o período de 2008 a 2011. Os autores observaram que a maioria das internações ocorreu entre 60 e 69 anos, sendo que o sexo masculino apresentou a maior taxa de internação nas faixas etárias de 60 a 69 e de 70 a 79 anos. As principais causas de internação foram as doenças dos aparelhos circulatório e respiratório, neoplasias, doenças do aparelho digestivo, infecciosas e parasitárias, lesões por envenenamento e causas externas. Esses fatos estão em consonância com os resultados encontrados para os Grupos I e III desta pesquisa.

Outro estudo 12 analisou a mortalidade e morbidade em idosos no Município de Maringá entre 1996 e 1998, no qual observou que as doenças circulatórias, neoplasias e respiratórias representaram $73 \%$ dos óbitos. Com relação às internações, $72,1 \%$ ocorreram devido às doenças circulatórias, respiratórias e digestivas. Os coeficientes de internação foram superiores aos de óbito (exceto para neoplasias) e maiores para os homens. Além disso, as doenças dos sistemas nervoso, geniturinário, respiratório, digestivo e causas externas apresentaram maior razão da hospitalização/óbito.

As principais causas de internações entre os idosos paranaenses também foram observadas em pesquisa 13 sobre a morbidade, tempo e custos de internações para a população idosa de Londrina. Mostrou-se que a pneumonia foi a principal causa em número de internações e em dias de permanência no hospital e a quarta em termos de custos pagos pelo SUS. Por sua vez, o infarto agudo do miocárdio e outras doenças isquêmicas do coração ficaram em primeiro lugar em valores pagos pelo sistema, e terceira, em número de internações e em dias de permanência.

Com relação à representatividade das despesas com neoplasias referentes às mulheres em Londrina e Maringá, sugere-se que esse resultado pode ser explicado pela existência de Centros de Referências para tratamento destas doenças nos municípios destacados e pela abrangência geográfica no atendimento. Londrina abriga uma Unidade de Assistência de Alta Complexidade em Oncologia (UNACOM) e um Centro de Assistência de Alta Complexidade em Oncologia (CACON) prestando atendimento às 17ạ, 18ạ, 19ạ e 20a Regionais de Saúde. Maringá, por sua vez, tem três Unidades de Assistência de Alta Complexidade em Oncologia (UNACOM), responsáveis pelas 13ạ, 14a e 15a Regionais de Saúde 39 .

Destaca-se que um dos aspectos preocupantes do envelhecimento é a doença oncológica. O câncer está presente em todas as etiologias, independentemente de classe social, cultura ou religião. A incidência da doença aumenta com o avanço da idade, que está associada a fatores de riscos para o desenvolvimento de tipos específicos de câncer e apresenta a menor eficácia dos mecanismos de reparação celular nos indivíduos com 60 anos ou mais. Os pacientes idosos com câncer, geralmente, apresentam comorbidades, reserva fisiológica restrita, limitações funcionais incapacidade física e outros agravos da idade 40 .

Ainda de acordo com os resultados desta pesquisa, constatou-se que os Grupos IV (regionais de saúde de Telêmaco Borba, Cianorte, Apucarana, Campo Mourão e Francisco Beltrão) e V (Foz do Iguaçu, Cascavel, Toledo, Pato Branco e Ponta Grossa) podem ser classificados no nível intermediário de desenvolvimento econômico e humano, pois apresentaram PIB per capita elevado, mas em um nível menor quando comparado às regiões I e III do Paraná. Além disso, tem níveis de IDH-M satisfatórios, pois o valor está próximo a 1 .

A associação entre fatores socioeconômicos e demográficos com morbidades e gastos hospitalares para o Grupo IV foi maior nas doenças do sistema nervoso (sexo masculino) e do aparelho circulatório (sexo feminino). É interessante ressaltar que nos Grupos I e III as doenças do sistema nervoso corresponderam à menor causa de internação e custos para ambos os sexos.

Resultados de estudo para o Paraná 38 indicam que as doenças do sistema nervoso representaram apenas $1,8 \%$ das 773.483 internações entre 2008 e 2011 . No entanto, as doenças relativas ao aparelho circulatório foram as mais prevalentes no estado $(28,7 \%)$ no mesmo período. Lo- 
go, a frequência das doenças do sistema nervoso apresentada na região IV indica a necessidade de maiores investigações nesta particularidade.

Para o Grupo V a influência dos fatores está relacionada, principalmente, às morbidades por doenças respiratórias para homens e mulheres. Contudo, foram as doenças do aparelho circulatório e do coração que demandaram o maior volume de recursos financeiros nesta área.

Os Grupos II (Paranavaí, Jacarezinho, Cornélio Procópio, União da Vitória, Umuarama, Guarapuava, Ivaiporã e Irati) e VI (Paranaguá) podem ser classificados como áreas menos desenvolvidas em termos de desenvolvimento humano quando comparadas às demais regiões do estado, pois os valores do IDH-M foram os mais baixos. No quesito PIB per capita há uma disparidade entre essas regiões, pois a primeira demonstra os menores valores e a segunda, o nível mais alto deste indicador no estado.

O destaque do Grupo VI no fator econômico pode estar relacionado à presença do Porto de Paranaguá, que é o segundo maior porto brasileiro em movimentação de cargas e maior complexo graneleiro da América Latina. É o principal canal de exportação de commodities agrícolas (soja e milho) produzidos no Brasil para o mercado internacional ${ }^{41}$. Assim, essa atividade portuária apresenta forte impacto na economia dessa região e explica o comportamento do indicador econômico.

Os resultados desta pesquisa mostraram que nos Grupos II e VI a relação entre os indicadores socioeconômicos e demográficos com as morbidades e custos hospitalares estiveram mais evidenciados nas doenças do aparelho circulatório e do coração para homens e mulheres. Esse fato está em consonância com o estudo desenvolvido por Carmo et al. 14 , que analisaram as principais causas de hospitalização em idosos dos municípios de Cândido de Abreu e Jardim Alegre (pertencentes à 22a Regional de Saúde, Ivaiporã), em 2005. Verificaram que existe alta prevalência de internamentos e de doenças dos aparelhos cardiovascular e respiratório; além disso, as patologias mais prevalentes em idosos foram doenças crônicas, que exigem acompanhamento constante e custoso para o SUS.

Em regiões com menor grau de desenvolvimento, os determinantes sociais como escolaridade e poder aquisitivo podem influenciar no perfil epidemiológico das doenças crônicas. De forma geral, há predominância de gradientes de morbidade desfavoráveis à população de pior nível socioeconômico, com exceção de algumas doenças, como o câncer, cujo gradiente se inverte, acometendo com maior frequência os segmentos mais favorecidos economicamente $42,43,44$.
Nessa linha, observa-se que o processo de envelhecimento gera a demanda por diversos serviços na área de saúde, sendo uma delas a internação hospitalar. Essa procura gera preocupação crescente do impacto desse processo no setor de saúde, pois o uso dos serviços de média e alta complexidades ofertados e dos equipamentos de alta tecnologia vem ocorrendo com maior intensidade e frequência com os idosos no Brasil. Assim, quanto maior a demanda por esses serviços, maior a ocupação dos leitos hospitalares com essa categoria da população, e com longas e sucessivas internações. Esse fato pode ser consequência da falta de acompanhamentos regulares pela atenção primária e outros serviços de saúde preventivos 45 .

Conforme observado neste estudo, a demanda dos idosos está centrada, principalmente, na utilização de serviços por doenças circulatórias, respiratórias e cardiovasculares, consideradas as particularidades socioeconômicas e demográficas destacadas. As patologias de base dessas doenças vêm associadas a outras complicações, o que aumenta o número de internações a esse público vulnerável às condições de saúde e os custos para o SUS 13. A utilização de recursos tecnológicos e de profissionais especializados encarece ainda mais os atendimentos oferecidos a essa população 45 .

Portanto, ao considerar os aspectos socioeconômicos e demográficos das regionais de saúde do Paraná, foi possível identificar as diferenças no comportamento das internações (dentre os cinco tipos de doenças analisadas) e dos custos hospitalares do SUS para cada agrupamento. Baseando-se na composição desse perfil epidemiológico, ações de prevenção e promoção de saúde aos idosos do Paraná podem ser realizadas com enfoque nos tipos de doenças e nas especificidades de desenvolvimento econômico e humano de cada área.

Os resultados desta pesquisa reforçam também a relevância do sistema de informações em saúde no Estado do Paraná e, por sua vez, no Brasil, como instrumento de elaboração, planejamento e monitoramento das ações de diagnóstico, prevenção e promoção à saúde direcionado à população idosa. O SUS pode desenvolver estratégias para ofertar um atendimento cada vez mais eficiente a essa parte da população que está em ampla expansão e demandando cada vez mais serviços de saúde de média e alta complexidades. 


\section{Colaboradores}

C. L. Kernkamp, C. K. F. Costa e E. M. Massuda contribuíram com a concepção do projeto de pesquisa, coleta, estimação e interpretação dos dados, redação do artigo, correções e aprovação final da versão a ser publicada. E. S. Silva colaborou na coleta, estimação, interpretação dos dados, redação do artigo e correções e aprovação final da versão a ser publicada. M. U. Yamaguchi e M. P. Bernuci participaram na interpretação dos dados, redação do artigo, correções e aprovação final da versão a ser publicada.

\section{Referências}

1. Carvalho JAM, Rodríguez-Wong LL. A transição da estrutura etária da população brasileira na primeira metade do século XXI. Cad Saúde Pública 2008; 24:597-605.

2. Moraes EN. Atenção à saúde do idoso: aspectos conceituais. Brasília: Organização Pan-Americana da Saúde; 2012.

3. Instituto Brasileiro de Geografia e Estatística. Dados preliminares do Censo 2010 já revelam mudanças na pirâmide etária brasileira. http://www.ibge. gov.br/ibgeteen/censo2010/index.html (acessado em 25/Mar/2013).

4. Michael JWP, Schluter-Brust KU, Eysel P. The epidemiology, etiology, diagnosis, and treatment of osteoarthritis of the knee. Dtsch Arztebl Int 2010; 107:152-62.

5. Camaro AA, organizador. Os novos idosos brasileiros: muito além dos 60. Rio de Janeiro: Instituto de Pesquisa Econômica Aplicada; 2004.

6. Nunes A. O envelhecimento populacional e as despesas do Sistema Único de Saúde. In: Camarano AA, organizador. Os novos idosos brasileiros: muito além dos 60. Rio de Janeiro: Instituto de Pesquisa Econômica Aplicada; 2004. p. 427-50.

7. Santos JS, Barros MDA. Idosos do Município do Recife, Estado de Pernambuco, Brasil: uma análise da morbimortalidade hospitalar. Epidemiol Serv Saúde 2008; 17:177-86.

8. Góis ALB, Veras RP. Informações sobre a morbidade hospitalar em idosos nas internações do Sistema Único de Saúde do Brasil. Ciênc Saúde Coletiva $2010 ; 15: 2859-69$

9. Loyola AIF. Causas de internações hospitalares entre idosos brasileiros no âmbito do Sistema Único de Saúde. Epidemiol Serv Saúde 2004; 13:229-38.

\section{Agradecimentos}

Ao Conselho Nacional de Desenvolvimento Científico Tecnológico (CNPq) pela Bolsa de Produtividade em Pesquisa à Dra. C. K. F. Costa.
10. Barreto ML, Carmo EH. Padrões de adoecimento e de morte da população brasileira: os renovados desafios para o Sistema Único de Saúde. Cienc Saúde Coletiva 2007; 12 Suppl:1779-90.

11. Peixoto SV, Giatti L, Afradique ME, Lima-Costa MF. Custo das internações hospitalares entre idosos brasileiros no âmbito do Sistema Único de Saúde. Epidemiol Serv Saúde 2004; 13:239-46.

12. Mathias TAF, Jorge MHP. Hospitalização e mortalidade em idosos: um exercício de análise comparativa. Ciênc Cuid Saúde 2005; 4:25-36.

13. Martin GB, Cordoni Júnior L, Bastos YGL, Silva PV. Assistência hospitalar à população idosa em cidade do Sul do Brasil. Epidemiol Serv Saúde 2006; 15:59-65.

14. Carmo CN, Hacon SS, Souza S, Jacobson LSV, Mourão DS, Ignotti E. Mortality due to cardiorespiratory diseases in elderly people in Mato Grosso state, 1986 to 2006. Rev Saúde Pública 2010; 44:1112-9.

15. Jobim EFC, Souza VO, Cabrera AS. Causas de hospitalização de idosos em dois hospitais gerais pelo Sistema Único de Saúde (SUS). Acta Sci Health Sci 2010; 32:79-83.

16. Faria AJA, Rosa JRM, Santos MW, Souza MB, Pinto RM, Picoli RP. Caracterização e situação de saúde do idoso na Região Metropolitana de São Paulo. http://observasaude.fundap.sp.gov.br/RgMetropolitana/idoso/Documents/Semin\%C3\%A1rio\%20 Sa\% C3\%BAde \% 20do\% 20 Id oso $\% 20$ na $\% 20$ regi\%C3\%A3o\%20metropolitana\%20de $\% 20$ S\%C3\%A3o\%20Paulo/SdIdsPROAHSA_2011jun08. docx (acessado em 23/Jun/2015). 
17. Oliveira MC, Rodrigues Neto JF, Silveira MF, Neves DMR, Vilhena JM, Oliveira JF, et al. Impacto dos fatores de risco para doenças crônicas não transmissíveis na qualidade de vida. Ciênc Saúde Coletiva 2013; 18:873-82.

18. Miller T, Castanheira HC. The fiscal impact of population aging in Brazil: 2005-2050. Rev Bras Estud Popul 2013; 30 Suppl:S5-23.

19. Kilsztajn S, Rossbach A, Câmara MB, Carmo MSN. Serviços de saúde, gastos e envelhecimento da população brasileira. Rev Bras Estud Popul 2003; 20:93-108.

20. Roy A, Roe MT, Neely ML, Cyr DD, Zamoryakhin D, Fox KA, et al. Impact of human development index on the profile and outcomes of patients with acute coronary syndrome. Heart 2015; 101:279-86.

21. Cobas RA, Ferraz MB, Matheus ASM, Tannus LRM, Silva ATK, Araujo LA, et al. Heterogeneity in the costs of type 1 diabetes in a developing country: what are the determining factors? Diabetol Metab Syndr 2013; 5:1-7.

22. Berenstein CK, Wajnman S. Efeitos da estrutura etária nos gastos com internação no Sistema Único de Saúde: uma análise de decomposição para duas áreas metropolitanas brasileiras. Cad Saúde Pública 2008; 24:2301-13.

23. Possoli S. Técnicas de análise multivariada para avaliação das condições de saúde dos municípios do Rio Grande do Sul - Brasil. Rev Saúde Pública 1984; 18:288-300.

24. Monteiro C, Silva B. A utilização de técnicas multivariadas na análise do posicionamento e segmentação de empresas do sistema suplementar de saúde. http://www.aedb.br/seget/artigos07/1324 servicos_fatorial\%20e\%20cluster.pdf (acessado em 14/Dez/2013).

25. Campanário P, Maia PB. Metodologia de correção e ajuste da mortalidade com tábuas-modelo de vida atualizadas para o Brasil. Epidemiol Serv Saúde 2004; 13:147-74.

26. Esquarcini R, Marques JM. Classificação dos municípios paranaenses segundo suas políticas setoriais pela análise multivariada. Revista da FAE 2006; 9:83-93.

27. Oliveira A, Amaral V. A análise fatorial de correspondências na investigação em psicologia: uma aplicação ao estudo das representações sociais do suicídio adolescente. Anál Psicol 2007; 25:271-93.

28. Peluso ETP, Blay SL. Percepção da depressão pela população da cidade de São Paulo. Rev Saúde Pública 2008; 42:41-8.

29. Freitas ED. Agrupamento de fatores de risco cardiovasculares em população rural, por meio da análise fatorial [Dissertação de Mestrado]. Belo Horizonte: Universidade Federal de Minas Gerais; 2008.

30. Costa MF. Análise fatorial confirmatória dos sintomas do transtorno de estresse pós-traumático em adultos assistidos pelo Programa Médico de Família de Niterói [Dissertação de Mestrado]. Niterói: Universidade Federal Fluminense; 2010.
31. Simberg ARF. Cluster de sintomas em adolescentes com câncer [Dissertação de Mestrado]. Guarulhos: Universidade Guarulhos; 2011.

32. Programa das Nações Unidas para o Desenvolvimento. O que é o IDHM? http://www.pnud.org.br/ idh/IDHM.aspx?indiceAccordion=0\&li=li_IDHM (acessado em 23/Jun/2015).

33. Everitt BS, Der GA. Handbook of statistical analyses using SAS. London: Chapman \& Hall; 1997.

34. Johson RA, Wichern DW. Applied multivariate statistical analysis. Englewood Cliffs: Prentice Hall; 1982.

35. Rencher A. Methods of multivariate analysis. 2nd Ed. New York: John Wiley \& Sons; 2002.

36. Reis E. Estatística multivariada aplicada. 2a Ed. Lisboa: Edições Silabo; 1997.

37. Silveira RE, Santos AS, Sousa MC, Monteiro TSA. Gastos relacionados a hospitalizações de idosos no Brasil: perspectivas de uma década. Einstein 2013; 11:514-20.

38. Castro VC, Borghi AC, Mariano PP, Fernandes CAM, Mathias TAF, Carreira L. Perfil de internações hospitalares de idosos no âmbito do Sistema Único de Saúde. Rev RENE 2013; 14:791-800.

39. Instituto Nacional de Câncer José de Alencar Gomes da Silva. Onde tratar pelo SUS. http://www. inca.gov.br/wps/wcm/connect/cancer/site/tra tamento/ondetratarsus/PR (acessado em 25/ Jun/2015).

40. Soares LC, Santana MG, Muniz RM. O fenômeno do câncer na vida de idosos. Ciênc Cuid Saúde 2010; 9:660-7.

41. Secretaria de Infraestrutura e Logística, Governo do Estado do Paraná. História do Porto de Paranaguá. http://www.portosdoparana.pr.gov.br/ modules/conteudo/conteudo.php?conteudo=26 (acessado em 23/Jun/2015).

42. Barros MB, Francisco PM, Zanchetta LM, César CL. Tendências das desigualdades sociais e demográficas na prevalência de doenças crônicas no Brasil, PNAD: 2003-2008. Ciênc Saúde Coletiva 2011; 16:3755-68.

43. Dalstra JAA, Kunst AE, Borrell C, Breeze E, Cambois E, Costa G, et al. Socioeconomic differences in the prevalence of common chronic diseases: an overview of eight European countries. Int J Epidemiol 2005; 34:316-26.

44. Barreto SM, Figueiredo RC. Doença crônica, autoavaliação de saúde e comportamento de risco: diferença de gênero. Rev Saúde Pública 2009; 43 Suppl 2:38-47.

45. Alfradique MA, Bonolo PF, Dourado I, Lima-Costa MF, Macinko J, Mendonça CS, et al. Internações por condições sensíveis à atenção primária: a construção da lista brasileira como ferramenta para medir o desempenho do sistema de saúde (Projeto ICSAP-Brasil). Cad Saúde Pública 2009; 25:1337-49. 


\section{Abstract}

Growth in the elderly population has increased both the demand for health services and healthcare expenses, with relevant consequences for economic stability. The current study aimed to analyze the morbidity profile and hospital expenses with elderly patients in relation to socioeconomic and demographic conditions in Paraná State, Brazil, from 2008 to 2012, applying principal components analysis and groupings. Regions with higher and lower economic and human development showed high prevalence of hospitalizations and costs pertaining to the circulatory and respiratory systems and cardiac diseases for males and females. In regions with intermediary development, diseases of the nervous system in men and of the circulatory system in women had the highest morbidity and hospital expenses. Thus, measures for prevention and health promotion in this elderly population should be targeted to regional investigations.

Demographic Aging; Aged; Hospitalization; Multivariate Analysis

\section{Resumen}

El aumento de la población anciana conlleva una creciente demanda de servicios de salud y expansión de gastos, con consecuencias relevantes sobre la estabilidad económica. En este contexto, el objetivo del estudio fue analizar el perfil de las morbilidades y gastos hospitalarios con ancianos en relación con las condiciones socioeconómicas y demográficas de Paraná, Brasil, durante el período de 2008 a 2012, aplicándose el análisis factorial en componentes principales y de agrupamientos. Las regiones con mayor y menor desarrollo económico y humano presentaron una alta prevalencia de los internamientos y de los costes relativos a las enfermedades del aparato circulatorio, respiratorio y del corazón para el sexo masculino y femenino. Ya en las regiones con un nivel de desarrollo intermedio, las enfermedades del sistema nervioso en hombres $y$ del aparato circulatorio en mujeres se destacaron respecto a las morbilidades y gastos hospitalarios. Así, acciones de prevención y promoción de salud para esa población anciana pueden ser dirigidas hacia investigaciones regionales.

Envejecimiento de la Población; Anciano;

Hospitalización; Análisis Multivariante
Recebido em 19/Mar/2015

Versão final reapresentada em 01/Jul/2015 Aprovado em 22/Fev/2016 